\title{
Significance of miR-27a and miR-31 in early diagnosis and prognosis of colorectal cancer
}

\author{
QINGJUN CUI \\ Department of Laboratory, Xintai People's Hospital, Xintai, Shandong 271200, P.R. China
}

Received January 21, 2019; Accepted May 10, 2019

DOI: $10.3892 / \mathrm{ol} .2019 .10621$

\begin{abstract}
Clinical significance of micro-ribonucleic acid (miR)-27a and miR-31 in the early diagnosis and prognosis of colorectal cancer were investigated. Forty patients with colorectal malignancy admitted to Xintai People's Hospital from February 2014 to April 2018 were enrolled as the observation group, of which 30 patients were diagnosed via pathological biopsy. Another 40 patients diagnosed with colorectal polyp and receiving surgical treatment were selected as the control group. The relative amount of miR-27a and miR-31 was measured. The relative expression levels of miR-27a and miR-31 in patients were analyzed. The diagnostic sensitivity, specificity and accordance rates of positive miR-27a and miR-31 expression in colorectal cancer were recorded. The correlation of the relative expression levels of miR-27a and miR-31 with the survival time of patients were analyzed. In the observation group, the relative expression levels of miR-27a and miR-31 in patients with lymph node metastasis and distant metastasis were higher than those in patients without lymph node metastasis and distant metastasis $(\mathrm{P}<0.05)$. Histological type of patients with non-mucinous carcinoma had increased relative expression levels of miR-27a and miR-31 in comparison with those with mucinous carcinoma $(\mathrm{P}<0.05)$. In terms of Duke's grade, the relative expression levels of miR-27a and miR-31 in patients with grade $C$ and $D$ were higher than those in patients with grade $\mathrm{A}$ and $\mathrm{B}(\mathrm{P}<0.05)$. The diagnostic sensitivity, specificity and accordance rate of positive miR-27a expression were lower than those of positive miR-31 expression. The relative expression levels of miR-27a and miR-31 were positively correlated with the survival time of patients $(\mathrm{P}<0.05)$. The expression levels of miR-27a and miR-31 are related to distant metastasis and tumor grade of patients with colorectal cancer, and positively associated with the survival time of patients, having high diagnostic value.
\end{abstract}

Correspondence to: Dr Qingjun Cui, Department of Laboratory, Xintai People's Hospital, 1329 Xinfu Road, Xintai, Shandong 271200, P.R. China

E-mail: gb5982r@163.com

Key words: miR-27a, miR-31, colon cancer, rectal cancer

\section{Introduction}

Colorectal cancer is a digestive system disease seriously threatening human health, it ranks 3rd in the world in terms of incidence and mortality rates (1). With the changes in lifestyle and diet customs in China, the incidence rate of colorectal cancer has increased significantly (2). Previous studies have proven that the pathogenesis of colorectal cancer is related to many factors (3-5). As a kind of endogenous non-coding single-stranded ribonucleic acid (RNA), microRNA (miRNA) can adjust the expression balance between proto-oncogenes and oncogenes, thereby affecting the proliferation, differentiation and apoptosis of malignant tumors and playing an important role in the occurrence and development of malignant tumors (6).

A current study on miRNA confirmed that miR-150 and miR-215 are associated with the incidence of colorectal cancer (7). However, research on the expression of miR-27a and miR-31 in colorectal cancer is rare, especially the value of miR-27a and miR-31 expression in the diagnosis of colorectal cancer and its association with the survival time of patients (8).

In this study, the expression levels of miR-27a and miR-31 and their early diagnostic significance in colorectal cancer were investigated, and the prognosis of patients was evaluated.

\section{Patients and methods}

General data. A total of 40 colorectal malignancy patients admitted to Xintai People's Hospital (Xintai, China) from February 2014 to April 2018 were selected as the observation group. Of these, 30 patients were diagnosed through pathological biopsy. A further 40 colorectal polyp patients receiving surgical treatment were enrolled as the control group. All enrolled patients signed informed consent on enrollment. Approval for the study was obtained from the Ethics Committee of Xintai People's Hospital. None of the patients enrolled had tumor-related treatment prior to enrollment. Patients with definite malignant tumors of other sites, with severe heart, lung, liver and kidney dysfunction, with mental disorder, undergoing gastrointestinal surgery previously, or with Karnofsky performance scale (KPS) score $>70$ points, were excluded.

Reagents and instruments. Reagents: TaqMan MicroRNA assay kit (provided by Applied Biosystems; Thermo Fisher Scientific, Inc., Waltham, MA, USA), and PrimeScript ${ }^{\mathrm{TM}}$ RT 
Table I. Comparison of the relative expression levels of miR-27a and miR-31 among patients with different data in observation group.

\begin{tabular}{|c|c|c|c|c|c|c|c|}
\hline Variable & $\mathrm{n}$ & $\operatorname{miR}-27 \mathrm{a}$ & $t$ value & P-value & miR-31 & $\mathrm{t}$ value & P-value \\
\hline Sex & & & 0.388 & 0.703 & & 0.017 & 0.986 \\
\hline Male & 23 & $0.0086 \pm 0.0009$ & & & $0.00625 \pm 0.0008$ & & \\
\hline Female & 17 & $0.0085 \pm 0.0009$ & & & $0.00621 \pm 0.0008$ & & \\
\hline Tumor site & & & 0.000 & 1.000 & & 0.000 & 1.000 \\
\hline Rectum & 16 & $0.0085 \pm 0.0009$ & & & $0.00623 \pm 0.0009$ & & \\
\hline Colon & 24 & $0.0085 \pm 0.0009$ & & & $0.00623 \pm 0.0008$ & & \\
\hline Lymph node metastasis & & & 4.307 & $<0.001$ & & 11.742 & $<0.001$ \\
\hline Yes & 23 & $0.0135 \pm 0.0008$ & & & $0.00836 \pm 0.0010$ & & \\
\hline No & 17 & $0.0063 \pm 0.0001$ & & & $0.00515 \pm 0.0006$ & & \\
\hline Distant metastasis & & & 31.929 & $<0.001$ & & 4.120 & $<0.001$ \\
\hline Yes & 12 & $0.0165 \pm 0.0011$ & & & $0.00716 \pm 0.0009$ & & \\
\hline No & 28 & $0.0059 \pm 0.0009$ & & & $0.00598 \pm 0.0008$ & & \\
\hline Histological type & & & & & & 3.907 & $<0.001$ \\
\hline Mucinous carcinoma & 7 & $0.0076 \pm 0.0010$ & & & $0.00601 \pm 0.0008$ & & \\
\hline Non-mucinous carcinoma & 33 & $0.0099 \pm 0.0008$ & & & $0.00656 \pm 0.0009$ & & \\
\hline Duke's grade & & & 49.602 & $<0.001$ & & 15.859 & $<0.001$ \\
\hline $\mathrm{A}$ and $\mathrm{B}$ & 30 & $0.0035 \pm 0.0010$ & & & $0.00413 \pm 0.0010$ & & \\
\hline C and D & 10 & $0.0212 \pm 0.0009$ & & & $0.00959 \pm 0.0007$ & & \\
\hline
\end{tabular}

reagent kit, TRIzol, fetal bovine serum and agar medium (Promega Corporation, Madison, WI, USA) and PCR kit (Jianlun Science and Technology Co., Guangdong, China) were used in the current study. Instruments used were: ultralow-temperature refrigerator (Sanyo, Osaka, Japan), 7500 HT real-time polymerase chain reaction (PCR) detector, and pure water and water purifier (Angel Water, Inc., Barrington, IL, USA).

Extraction of RNA. First, $200 \mu \mathrm{l}$ blood gas sample was collected and refrigerated, followed by thawing at room temperature and shaking. Then, TRIzoI LS solution was added into the sample three times, shaken well, placed at room temperature for $10 \mathrm{~min}$ and denatured at room temperature. Next, the sample was added with chloroform, mixed on a shaker, and put aside at room temperature for $15 \mathrm{~min}$. Thereafter, RNA was extracted after centrifugation at $1,000 \mathrm{x} \mathrm{g}$ and $40^{\circ} \mathrm{C}$ for $15 \mathrm{~min}$, and the supernatant was collected and added with 1.5-fold $95 \%$ ethanol. miRNeasy was used as a manual to carry out RNA enrichment.

Measurement of serum miR-27a and miR-31 expression levels. Enzyme-linked immunosorbent assay was applied to measure serum miR-27a and miR-31 expression levels. Target RNA $(0.5 \mu \mathrm{g})$ was taken and subjected to reverse transcription at $37^{\circ} \mathrm{C}$ and $85^{\circ} \mathrm{C}$ for $1 \mathrm{~h}$ and 5 min to complementary deoxyribonucleic acid (cDNA), respectively. cDNA $(0.5 \mu \mathrm{l})$ (product of reverse transcription) was obtained and used for SYBR-Green qPCR (Thermo Fisher Scientific, Inc.). All the operations were conducted by a tester with more than 5 years of laboratory experience in strict accordance with the instructions. The reaction conditions were: $95^{\circ} \mathrm{C}$ for $10 \mathrm{~min}, 95^{\circ} \mathrm{C}$ for $10 \mathrm{sec}$, $60^{\circ} \mathrm{C}$ for $30 \mathrm{sec}, 72^{\circ} \mathrm{C}$ for $10 \mathrm{sec}$, continuously performed for
40 cycles. The cycle threshold $(\mathrm{Cq})$ values of all samples were recorded and expressed by the $2^{-\Delta \mathrm{Cq}}$ method (9), wherein $\Delta \mathrm{Cq}$ is the difference between $\mathrm{Cq}_{(\mathrm{miR}-27 \mathrm{a} \text { or miR-31) }}$ and $\mathrm{Cq}_{\mathrm{U} 6}$. $\beta$-actin was used as internal reference. Primer sequences were as follows: miR-27a, forward, 5'-TGCGTTCAGTGGCTAAG-3' and reverse, 5'-CTCAACTGGTGTCGTGGA-3'; miR-31, forward, 5'-ACGGCAAGATGCTGGGCA-3' and reverse, 5'-CAGTGCTGGTCCGAGTGA-3'.

Evaluation of miR-27a and miR-31 positive results. The positive signal was based on the collection of living tissue samples, and qPCR was carried out. The cytoplasm of sample cells was mainly observed. The results were evaluated based on color development of samples, in which strongly positive: brown or blue-black, moderately positive: yellow or blue, weakly positive: light yellow or light blue, and negative: unlimited color.

Observation indicators. The relative expression levels of miR-27a and miR-31 in patients with different data in the observation group were measured. The change tendencies in relative expression levels of miR-27a and miR-31 in two groups at different time points [at enrollment, before surgery, at 3 days after surgery and after chemotherapy (at discharge)] were determined. The diagnostic sensitivity, specificity and accordance rates of positive miR-27a and miR-31 expression in colorectal cancer were detected. Finally, the correlations of miR-27a and miR-31 expression levels with the survival time of patients were analyzed.

Statistical analysis. Statistical Product and Service Solutions (SPSS) 13.0 software (IBM Corp., Armonk, NY, USA) was used for data processing. Measurement data were expressed 
Table II. Comparison of positive miR-27a expression and pathological findings.

\begin{tabular}{lccr}
\hline \multirow{2}{*}{$\begin{array}{l}\text { miR-27a } \\
\text { expression }\end{array}$} & \multicolumn{2}{c}{ Pathological findings } & \\
\cline { 2 - 2 } & Positive & Negative & Total \\
\hline Positive & 27 & 4 & 31 \\
Negative & 3 & 6 & 9 \\
Total & 30 & 10 & 40 \\
\hline
\end{tabular}

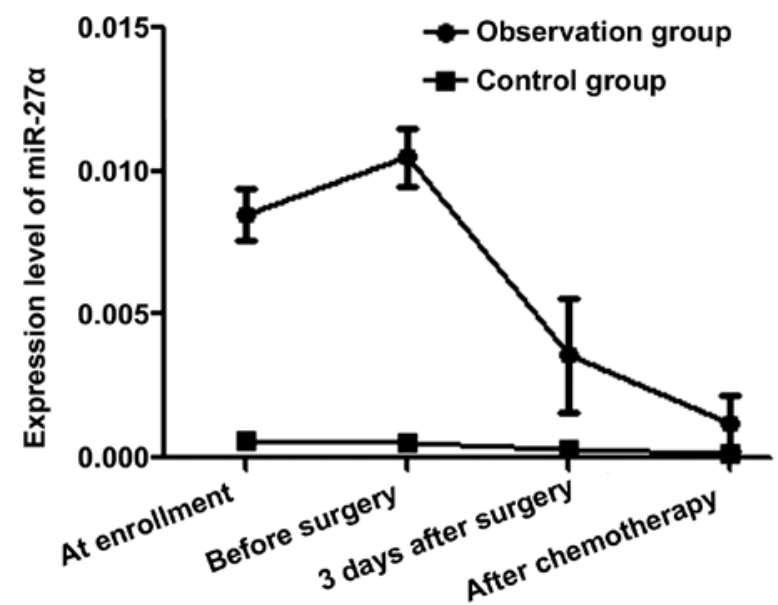

Figure 1. Changed trend in relative expression level of miR-27a in two groups at different time points. The relative expression level of miR-27a in observation group is clearly higher than that in control group at enrollment, before surgery, at 3 days after surgery and after chemotherapy $(\mathrm{P}<0.05)$.

as mean \pm standard deviation (mean $\pm \mathrm{SD}$ ). The t-test was employed for comparison of the mean between two groups, and $\chi^{2}$ test was used for comparison of rate between groups. Pearson's correlation was used for correlation analysis. $\mathrm{P}<0.05$ was considered to indicate a statistically significant difference.

\section{Results}

Comparison of the relative expression levels of miR-27a and miR-31 among patients with different data in observation group. Among the 40 patients in observation group, those with lymph node metastasis and distant metastasis had enhanced relative expression levels of miR-27a and miR-31 in comparison with those without lymph node metastasis and distant metastasis $(\mathrm{P}<0.05)$. In histological types, the relative expression levels of miR-27a and miR-31 in patients with non-mucinous carcinoma were higher than those in patients with mucous carcinoma $(\mathrm{P}<0.05)$. In Duke's grades, the relative expression levels of miR-27a and miR-31 in patients with grade $C$ and $D$ were higher than those in patients with grade $\mathrm{A}$ and $\mathrm{B}(\mathrm{P}<0.05)$ (Table I).

Changed trend in relative expression level of miR-27a in two groups at different time points. At enrollment, before surgery, at 3 days after surgery and after chemotherapy, the relative expression level of miR-27a was $0.0085 \pm 0.0009$, $0.0105 \pm 0.0010,0.0036 \pm 0.002$ and $0.0012 \pm 0.001$, respectively, in observation group, and $0.0006 \pm 0.0001,0.0005 \pm 0.0001$,
Table III. Comparison of positive miR-31 expression and pathological findings.

\begin{tabular}{lccr}
\hline \multirow{2}{*}{$\begin{array}{l}\text { miR-31 } \\
\text { expression }\end{array}$} & Pathological findings & \\
\cline { 2 - 3 } & Positive & Negative & Total \\
\hline Positive & 28 & 3 & 31 \\
Negative & 2 & 7 & 9 \\
Total & 30 & 10 & 40 \\
\hline
\end{tabular}

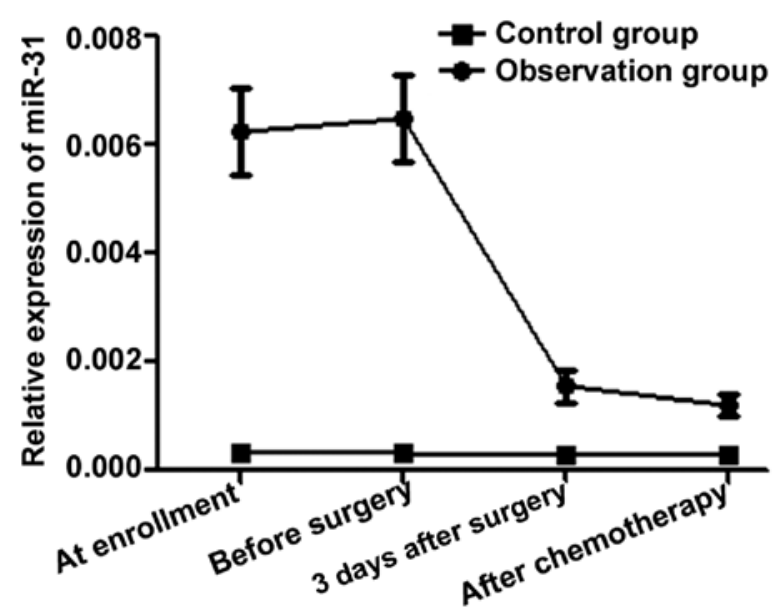

Figure 2. Changed tendency in relative expression level of miR-31 in two groups at different time points. The relative expression level of miR-31 in observation group is evidently higher than that in control group at enrollment, before surgery, at 3 days after surgery and after chemotherapy $(\mathrm{P}<0.05)$.

$0.0003 \pm 0.0001$ and $0.0002 \pm 0.0001$, respectively, in control group. Observation group had an evidently higher relative expression level of miR-27a than control group at enrollment, before surgery, at 3 days after surgery and after chemotherapy $(\mathrm{t}=55.176,62.932,10.422$ and $6.293, \mathrm{P}<0.001<0.05)($ Fig. 1$)$

Changed tendency in relative expression level of miR-31 in two groups at different time points. In observation group, the relative expression level of miR-31 was $0.00623 \pm 0.0008$, $0.00649 \pm 0.0008,0.00156 \pm 0.0003$ and $0.00119 \pm 0.0002$ at enrollment, before surgery, at 3 days after surgery and after chemotherapy, respectively. In the control group, the relative expression level of miR-31 was $0.00032 \pm 0.0001$ at enrollment, $0.00031 \pm 0.0001$ before surgery, $0.00029 \pm 0.0001$ at 3 days after surgery and $0.00030 \pm 0.0001$ after chemotherapy. The relative expression level of miR-31 was clearly increased in observation group compared with that in control group at enrollment, before surgery, at 3 days after surgery and after chemotherapy $(\mathrm{t}=46.362,48.480,0.963$ and 6.982, $\mathrm{P}<0.001<0.05)($ Fig. 2).

Comparison of positive miR-27a expression and pathological findings. The diagnostic sensitivity of positive miR-27a expression was $90.0 \%$, the diagnostic specificity was $60.0 \%$, and the diagnostic accordance rate was $82.5 \%$ (Table II).

Comparison of positive miR-31 expression and pathological findings. The diagnostic sensitivity, specificity and accordance 


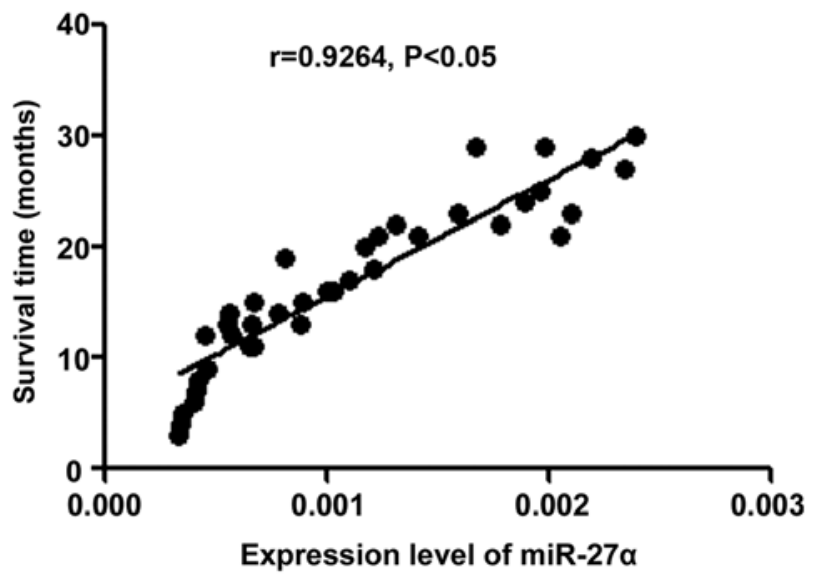

Figure 3. Correlation between the relative expression level of miR-27a and the survival time of patients by Pearson's correlation analysis. The relative expression level of miR-27a is positively correlated with the survival time of patients $(\mathrm{P}<0.05)$.

rate of positive miR-31 expression were $93.3 \%, 70.0 \%$, and $87.5 \%$, respectively (Table III).

Correlation between the relative expression level of $m i R-27 a$ and the survival time of patients. Pearson's correlation analysis showed that the relative expression level of miR-27a had a positive correlation with the survival time of patients $(\mathrm{r}=0.9264, \mathrm{P}<0.001<0.05)$ (Fig. 3).

Correlation between the relative expression level of $\mathrm{miR}-31$ and the survival time of patients. Pearson's correlation analysis showed that there was a positive relation between the relative expression level of miR-31 and the survival time of patients $(\mathrm{r}=0.9415, \mathrm{P}<0.001<0.05)$ (Fig. 4).

\section{Discussion}

Colorectal cancer is the most common malignant tumor of the digestive tract. The key is early diagnosis and treatment, and surgical resection is the most effective method (10). However, many patients are already in the advanced stage or do not have the opportunity to accept and undergo surgical treatment owing to lack of obvious clinical manifestations at the early stage, and no evidence of specific detection screening indicators (11). Therefore, an effective method for early diagnosis of colorectal cancer is imperative, which is preferably a non-invasive method with good reproducibility, specificity and sensitivity (12). The detection of plasma miRNA (an index relating to early diagnosis and prognosis evaluation of non-invasive tumors) has been widely used in clinical practice in recent years and successfully applied to the diagnosis, treatment and prevention evaluation of various malignant tumors (13). miRNAs are single-stranded non-coding RNAs, which are capable of specifically recognizing the 3'-untranslated region of target mRNAs, thereby promoting the degradation and reverse transcription of target mRNAs (14). Various studies have shown that miRNAs are associated with the occurrence and development of many malignancies (15-17).

In this study, patients with benign and malignant colorectal tumors were studied, and the expression levels of miR-27a and miR-31 were analyzed. Based on analyses of relative

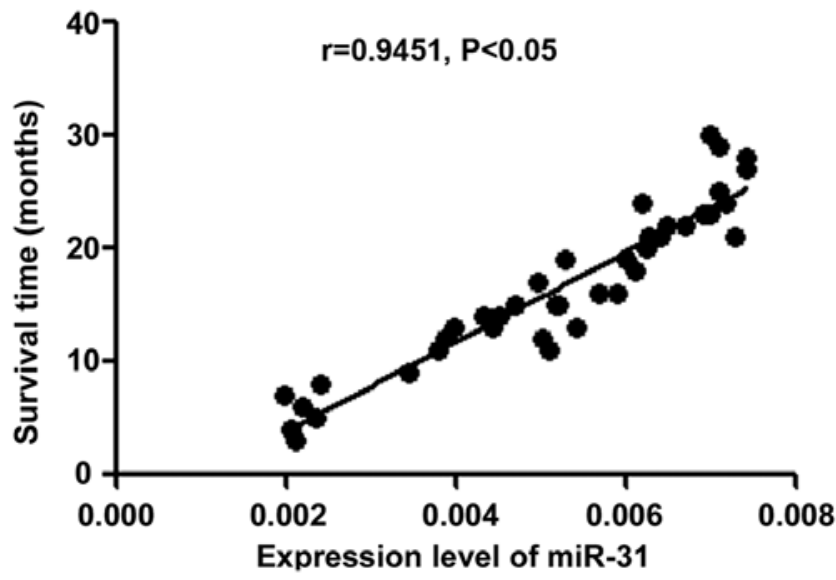

Figure 4. Relation between the relative expression level of miR-31 and the survival time of patients by Pearson's correlation analysis. The relative expression level of miR-31 is positively related to the survival time of patients $(\mathrm{P}<0.05)$.

expression levels of miR-27a and miR-31 in 40 patients with different data in observation group, patients with lymph node metastasis, distant metastasis, non-mucinous carcinoma (histological type) and Duke's grade C and D had overtly elevated relative expression levels of miR-27a and miR-31, suggesting that the expression levels of miR-27a and miR-31 are associated with tumor development, tumor grade and histological type of patients with colorectal cancer. In addition, analyses on changed tendencies of the relative expression levels of miR-27a and miR-31 in two groups at different time points (at admission, before surgery, at 3 days after surgery and after chemotherapy or at discharge) revealed that miR-27a and miR-31 expression levels in observation group were distinctly higher than those in control group, indicating that the expression levels of miR-27a and miR-31 are correlated with the occurrence of colorectal cancer to some extent. Moreover, the positive expression of miR-27a and miR-31 and the pathological findings were compared, and it was found that the diagnostic sensitivity and specificity of positive miR-27a and miR-31 expression were over $90 \%$ and approximately $70.0 \%$, respectively, and the diagnostic accordance rates were close to $90 \%$. The above results indicate that miR-27a and miR-31 detection has guiding value for the diagnosis of colorectal cancer. Finally, the correlation analyses of the relative expression of miR-27a and miR-31 with the survival time of patients discovered that the relative expression levels of miR-27a and miR-31 were positively correlated with the survival time of patients, further suggesting that the relative expression levels of miR-27a and miR-31 in colorectal cancer patients are related to the survival time of patients. The lower the relative expression levels of miR-27a and miR-31 are, the longer the survival time of patients will be.

miR-31 regulates the levels of large tumor suppressor homolog 2 and protein phosphatase 2 A subunit B isoform R2- $\alpha$, thus accelerating the growth of malignant cells, and it also blocks the increase in levels of hypoxia-inducible factors (18), thereby leading to the proliferation of malignant cells. In particular, during the occurrence and development of colorectal cancer, miR-31 can activate the RAS signal transduction pathway in the body and inhibit the translation of Rasa-1, thus causing the proliferation of colorectal cancer cells (19). Moreover, 
the overexpression of miR-31 may result in distant metastasis of malignant tumor cells, so the level of miR-31 in the blood of patients with colorectal cancer is obviously increased. In addition, it is confirmed that miR-27a is highly expressed in tissues of a variety of malignant tumors including pancreatic cancer, breast cancer, thyroid cancer and ovarian cancer (20). The possible mechanism is that the overexpression of miR-27a reduces the protein level of B-cell translocation gene 2, inhibits the Ras/MEK/ERK pathway in colorectal cancer cells and thus increases c-Myc levels, and it downregulates expression levels of Golgi protein 73 and other tumor suppressor genes (21).

In conclusion, the expression levels of miR-27a and miR-31 are associated with distant metastasis and tumor grade of patients with colorectal cancer, having high diagnostic value and their expression levels are positively associated with the survival time of patients.

\section{Acknowledgements}

Not applicable.

\section{Funding}

No funding was received.

\section{Availability of data and materials}

The datasets used and/or analyzed during the current study are available from the corresponding author on reasonable request.

\section{Authors' contributions}

QC wrote the manuscript, performed the enzyme-linked immunosorbent assay, contributed to the conception, the design and the interpretation of the data of the study. QC read and approved the final manuscript.

\section{Ethics approval and consent to participate}

The study was approved by the Ethics Committee of Xintai People's Hospital (Xintai, China) and informed consents were signed by the patients or the guardians.

\section{Patient consent for publication}

Not applicable.

\section{Competing interests}

The authors declare that they have no competing interests.

\section{References}

1. Lv ZC, Fan YS, Chen HB and Zhao DW: Investigation of microRNA-155 as a serum diagnostic and prognostic biomarker for colorectal cancer. Tumour Biol 36: 1619-1625, 2015.

2. Eslamizadeh S, Heidari M, Agah S, Faghihloo E, Ghazi H, Mirzaei A and Akbari A: The role of microRNA signature as diagnostic biomarkers in different clinical stages of colorectal cancer. Cell J 20: 220-230, 2018

3. Fabian P and Němeček R: Prediction of EGFR blockade responses in metastatic colorectal carcinoma. Cesk Patol 54: 17-21, 2018 (In Czech).
4. Ramon L, David C, Fontaine K, Lallet E, Marcaillou C, MartinLannerée S, Decaulne V, Vazart C, Gélibert AH, Abdelali RB, et al: Technical validation of a reverse-transcription quantitative polymerase chain reaction in vitro diagnostic test for the determination of miR-31-3p expression levels in formalinfixed paraffin-embedded metastatic colorectal cancer tumor specimens. Biomark Insights 13: 1177271918763357, 2018.

5. Heublein S, Albertsmeier M, Pfeifer D, Loehrs L, Bazhin AV, Kirchner T, Werner J, Neumann J and Angele MK: Association of differential miRNA expression with hepatic vs. peritoneal metastatic spread in colorectal cancer. BMC Cancer 18: 201, 2018.

6. Bandres E, Agirre X, Bitarte N, Ramirez N, Zarate R, Roman-Gomez J, Prosper F and Garcia-Foncillas J: Epigenetic regulation of microRNA expression in colorectal cancer. Int J Cancer 125: 2737-2743, 2009.

7. Lundberg IV, Wikberg ML, Ljuslinder I, Li X, Myte R, Zingmark C, Löfgren-Burström A, Edin S and Palmqvist R: MicroRNA expression in KRAS- and BRAF-mutated colorectal cancers. Anticancer Res 38: 677-683, 2018.

8. Pugh S, Thiébaut R, Bridgewater J, Grisoni ML, Moutasim K, Rousseau F, Thomas GJ, Griffiths G, Liebaert F, Primrose J, et al: Association between miR-31-3p expression and cetuximab efficacy in patients with KRAS wild-type metastatic colorectal cancer: A post-hoc analysis of the New EPOC trial. Oncotarget 8: 93856-93866, 2017.

9. Livak KJ and Schmittgen TD: Analysis of relative gene expression data using real-time quantitative PCR and the 2(-Delta Delta C(T)) method. Methods 25: 402-408, 2001.

10. Wang YN, Chen ZH and Chen WC: Novel circulating microRNAs expression profile in colon cancer: A pilot study. Eur J Med Res 22: 51, 2017.

11. Takahashi H, Takahashi M, Ohnuma S, Unno M, Yoshino Y, Ouchi K, Takahashi S, Yamada Y, Shimodaira H and Ishioka C: microRNA-193a-3p is specifically down-regulated and acts as a tumor suppressor in BRAF-mutated colorectal cancer. BMC Cancer 17: 723, 2017.

12. Li J, Li X, Cen C, Ai X, Lin C and Hu G: The long non-coding RNA ENST00000547547 reduces 5-fluorouracil resistance of colorectal cancer cells via competitive binding to microRNA-31. Oncol Rep 39: 217-226, 2018.

13. Tian Y, Ma X, Lv C, Sheng X, Li X, Zhao R, Song Y, Andl T, Plikus MV, Sun J, et al: Stress responsive miR-31 is a major modulator of mouse intestinal stem cells during regeneration and tumorigenesis. eLife 6: 538-540, 2017.

14. Wang H, Luo J, Liu C, Niu H, Wang J, Liu Q, Zhao Z, Xu H, Ding Y, Sun J, et al: Investigating microRNA and transcription factor co-regulatory networks in colorectal cancer. BMC Bioinformatics 18: 388, 2017.

15. Yuan Z, Baker K, Redman MW, Wang L, Adams SV, Yu M, Dickinson B, Makar K, Ulrich N, Böhm J, et al: Dynamic plasma microRNAs are biomarkers for prognosis and early detection of recurrence in colorectal cancer. Br J Cancer 117: 1202-1210, 2017.

16. Liu X, Pan B, Sun L, Chen X, Zeng K, Hu X, Xu T, Xu M and Wang S: Circulating exosomal miR-27a and miR-130a act as novel diagnostic and prognostic biomarkers of colorectal cancer. Cancer Epidemiol Biomarkers Prev 27: 746-754, 2018.

17. Liang J, Tang J, Shi H, Li H, Zhen T, Duan J, Kang L, Zhang F, Dong $\mathrm{Y}$ and Han A: miR-27a-3p targeting RXR $\alpha$ promotes colorectal cancer progression by activating $\mathrm{Wnt} / \beta$-catenin pathway. Oncotarget 8: 82991-83008, 2017.

18. Chen M, Fang W, Wu X, Bian S, Chen G, Lu L and Weng Y: Distinct effects of rs895819 on risk of different cancers: An update meta-analysis. Oncotarget 8: 75336-75349, 2017.

19. Xu Q, Tong JL, Zhang CP, Xiao Q, Lin XL and Xiao XY: miR-27a induced by colon cancer cells in HLECs promotes lymphangiogenesis by targeting SMAD4. PLoS One 12: e0186718, 2017.

20. Ba S, Xuan Y, Long ZW, Chen HY and Zheng SS: MicroRNA-27a promotes the proliferation and invasiveness of colon cancer cells by targeting SFRP1 through the Wnt $/ \beta$-catenin signaling pathway. Cell Physiol Biochem 42: 1920-1933, 2017.

21. Zhao Y, Ting KK, Li J, Cogger VC, Chen J, Johansson-Percival A, Ngiow SF, Holst J, Grau G, Goel S, et al: Targeting vascular endothelial-cadherin in tumor-associated blood vessels promotes T-cell-mediated immunotherapy. Cancer Res 77: 4434-4447, 2017.

This work is licensed under a Creative Commons Attribution-NonCommercial-NoDerivatives 4.0 International (CC BY-NC-ND 4.0) License. 